\title{
Traduire pour transmettre: le cas des textes amérindiens.
}

\author{
Danielle Cyr \& Alexandre Sévigny \\ Université York \& Université McMaster
}

This article explores two sorts of problems that postcolonial amerindian translation poses: grammatical facts and socio-cultural context. In a first section, we discuss specific grammatical facts occurring in some First Nations languages that pose difficulties to the translator. In a second section, we discuss the process of translation of amerindian texts, concentrating on who is doing the translating and the importance of translation to the survival of endangered amerindian languages. All discussion is framed by the fact that amerindian languages are currently situated in amerindian cultures which are the product of colonial influences that shaped the socio-historical context within which amerindian languages evolved. These problems are discussed with specific reference to Migmaq, Innu and Montagnais, all endangered amerindian languages spoken in Canada and the United States. The target languages for the translations are either French or English.

\section{Introduction}

Le présent article a pour but de donner une vision "intérieure" de quelques questions et problèmes de traduction liés aux langues amérindiennes. Les langues amérindiennes sont, dans plusieurs sens, le produit d'une co-existence avec la langue de la puissance coloniale qui les domine. A part la difficulté créée par un climat de méfiance qui résulte de la colonisation, il y a aussi le fait que le maintien des langues amérindiennes est souvent rattaché à la langue du colonisateur comme médium de transmission de la langue amérindienne à préserver. Cette constatation a comme conséquence que tout enseignement de la langue amérindienne finit par être une traduction, une création de la part du traducteur. En fait, une sorte de réinterprétation à travers la lentille de la langue ou de la culture du colonisateur. Avant d'aborder l'examen des oeuvres traduites et le discours qui les entoure, nous ferons un tour d'horizon de ce que nous appelons l'intraduisible algonquien. Par cette expression, nous faisons référence à diverses catégories grammaticales et stylistiques qui reflètent et configurent la vision du monde algonquien ${ }^{1}$. On ne trouve que difficilement des équivalents dans les langues officielles que sont l'anglais et le français. Non seulement ces catégories échappent souvent aux traducteurs, mais, même lorsque ceux-ci en sont conscients, elles échappent d'une certaine manière à la traduction de sorte que, lorsqu'un texte algonquien est traduit en français ou en anglais, la vision amérindienne du monde peut s'en trouver substantiellement affectée. Si la seule identification de ces catégories pose problème au traducteur, la traduction à proprement parler en pose encore davantage. Ainsi, lorsqu'un texte algonquien est tra- 
duit, il perd généralement de sa finesse grammaticale et stylistique ainsi que la vision intérieure et sociétale du monde qu'il transporte intrinsèquement dans la langue originale. Nous utiliserons des exemples tirés de l'innu, du montagnais, du migmaq, du français et de l'anglais. Les exemples montagnais seront traduits vers le français, les autres concernent des traductions vers l'anglais.

Au Canada, on traduit aussi bien des langues autochtones vers l'anglais ou le français que d'une de ces deux langues officielles vers les langues autochtones. Dans le premier cas, il s'agit presque toujours de textes à caractère ethnologique, soit des contes et des légendes qui ont été d'abord enregistrés sur bandes magnétiques, puis transcrits verbatim et enfin traduits dans l'une des langues officielles. Ces textes sont la plupart du temps le produit de coopérations entre des chercheurs universitaires blancs et des consultants autochtones. En général, le texte traduit est fidèle à la langue d'arrivée, soit l'anglais ou le français. Dans le second cas, le texte est traduit du français ou de l'anglais en langue autochtone. Il s'agit presque toujours de textes gouvernementaux, de matériau pédagogique, ou de textes religieux (bible, prières chrétiennes, chants religieux, etc.). Généralement, le produit traduit est assez fidèle à la langue de départ, qu'il s'agisse du français ou de l'anglais mais le texte d'arrivée est d'un contenu souvent si inusité pour le lecteur autochtone, que ce dernier ne s'y reconnaît que partiellement du point de vue culturel.

\section{L'intraduisible: les faits de langue}

Dans cette section, nous discutons quelques traits des langues algonquiennes qui sont très difficiles à traduire en français ou en anglais.

\section{1. "Tu" comme première personne en grammaire: rôle social de la langue}

Les langues algonquiennes ne connaissent pas, à proprement parler, de voix passive. Elles possèdent cependant une forme grammaticale appelée la "direction", ou la "hiérarchie personnelle", et qui sert à exprimer les relations interpersonnelles souvent véhiculées par la voix passive dans d'autres langues.

Le terme "hiérarchie personnelle" signifie que dans les langues algonquiennes il existe une préséance de certaines personnes sur d'autres et que cette préséance s'exprime morphologiquement. Prenons le cas où je et $t u$ sont en interrelation, comme en français dans "je te vois" ou "tu me vois", par exemple. En montagnais, peu importe qui agit sur qui, il faut obligatoirement exprimer le $t u$ en premier. Autrement dit, dans l'expression des relations interpersonnelles $t u$ a toujours préséance sur je. Voyons quelques exemples: 
(1) Tshi-uâpam-in

2 - voir - 1 s

"Tu me vois"

Cet exemple exprime une relation directe de toi sur moi, ce qui est permis par la grammaire de la hiérarchie personnelle où tu a la possibilité d'agir sur je. Cependant, selon cette hiérarchie, il n'est guère possible d'exprimer directement l'action de je sur $t u$. La seule façon de faire de je le sujet grammatical du verbe exprimant l'action est d'indiquer une inversion du processus, inversion qui sera marquée formellement par un morphème appelé inversif (inv.), comme dans l'exemple suivant:

(2) Tshi-uâpam-it-in

2 - voir-inv- 1 s

"Tu es vu par moi" (= Je te vois)

Cette forme est beaucoup plus qu'une formule de politesse ou une forme passive, puisqu'il n'y a pas d'autre façon d'exprimer la chose dans cette langue. Il en va de même des relations entre je ou tu et $i l$. La première et la deuxième personne ont toujours préséance sur la troisième. On peut exprimer directement:

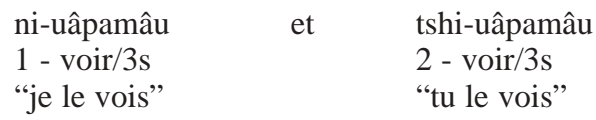

Mais pour dire "il me voit" ou "il te voit", on est obligé d'utiliser un morphème inversif de troisième personne, comme dans l'exemple suivant:

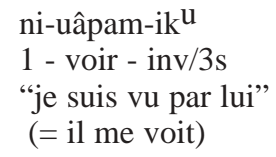
et tshi-uâpm-ik ${ }^{\mathrm{u}}$
2 - voir - inv/3s
"tu es vu par lui"
(= il te voit)

Malgré cette architecture conceptuelle et formelle, les grammairiens des langues algonquiennes, fidèles à la tradition indo-européenne, en sont venus à représenter dans les paradigmes verbaux les personnes verbales ${ }^{2}$ sujets de l'algonquien de la manière suivante: $\mathrm{je}=1$, tu=2 et $\mathrm{il}=3$. Cependant, $\mathrm{Cyr}$ (1996) montre que dans les langues algonquiennes ce n'est pas je qui est conçu comme première personne en grammaire, mais plutôt $t u$. Les indices de cette conceptualisation différente de la nôtre sont les suivants:

- les possessions inaliénables sont spontanément énoncées à la forme tu;

- la personne verbale tu a préséance sur la personne je;

- il faut un morphème inversif pour énoncer l'intervention de je sur tu;

- les locuteurs déclinent les paradigmes verbaux en commençant spontanément par la personne $t u$; 
- les enseignants de langue demandent aux grammairiens de mettre la personne $t u$ en tête des paradigmes verbaux.

Cette conceptualisation des paradigmes grammaticaux au niveau de la langue se répercute au niveau de la parole algonquienne dans l'importance accordée à l'interlocuteur dans la construction du discours oral. Autrement dit, l'importance accordée à $t u$ en grammaire est probablement le reflet de l'importance accordée à autrui comme partenaire dans la société. La langue est ici le véhicule et le miroir des rôles sociaux. Cependant, ces rôles et leur dynamisme ne transparaissent pas nécessairement au niveau de la traduction. Ils ne peuvent alors que se perdre dans le passage d'une langue algonquienne à une langue indo-européenne.

\subsection{L'obviatif: rôle textuel et conceptuel}

L'obviation (obv.) est une catégorie grammaticale [marque grammaticale] propre aux langues algonquiennes et dont la marque s'exprime autant par le verbe que par le nom et ses déterminants. D'un point de vue sémantique, on peut décrire cette catégorie comme l'expression de la "prédominance" d'un actant de troisième personne sur un autre actant de troisième personne. En linguistique algonquienne on désigne également cette catégorie "quatrième personne". Les termes anglais de proximation et de personal distance sont à ce propos révélateurs. Il convient cependant de prendre ces termes dans leur sens figuré et non pas dans le sens de 'distance physique'. L'actant sujet ou objet qui porte la marque de l'obviatif doit être perçu comme moins "saillant" que celui qui n'est pas obviatisé. Ce dernier, au contraire, apparaît par opposition comme mis en relief.

Peut-être l'obviation est-elle plus facile à comprendre si l'on examine d'abord sa fonction grammaticale et ses formes. En innu-montagnais, la marque de l'obviatif consiste à ajouter au radical le suffixe - $a$ pour les animés et le suffixe -nu pour les non-animés. Lorsque l'animé est obviatisé on ne peut plus faire la distinction entre singulier et pluriel. Par exemple uapmeu mashkua signifiera tout autant 'il voit un ours' que 'il voit des ours' . Lorsque le sujet d'un verbe est "obviatisé", l'accord se fait avec le verbe qui lui aussi porte la marque de l'obviatif constituée de différents morphèmes selon le mode et le temps du verbe.

Dans une proposition où il $\mathrm{y}$ a deux troisièmes personnes, par exemple "l'enfant voit le lièvre", une de ces troisièmes personnes devra obligatoirement être obviatisée, c'est-à-dire "éloignée mentalement", à l'arrièreplan, alors que l'autre, celle qui n'est pas marquée par un morphème d'obviatif, paraîtra par contraste comme plus visible, plus importante dans la trame du récit. En général, l'actant-sujet (nom ou pronom) est présenté comme plus important et l'actant-objet (nom ou pronom) comme moins important. Mais l' importance accordée aux participants relève avant tout du narrateur: il a toute liberté, selon ce qu'il veut mettre en évidence, de mar- 
quer l'objet comme prédominant et le sujet comme "éloigné”. Les exemples suivants (tirés de McNulty) illustrent le phénomène:

\begin{tabular}{|c|c|c|c|c|}
\hline $\begin{array}{l}\text { Kue } \\
\text { et } \\
\text { "Et les }\end{array}$ & $\begin{array}{l}\text { nâtshât } \\
\text { ils-vont-le-voir } \\
\text { enfants vont voir }\end{array}$ & $\begin{array}{l}\text { ntshent } \\
\text { les } \\
\text { le lièvre" }\end{array}$ & $\begin{array}{c}\text { auâssat } \\
\text { enfants } \\
\text { (McNulty }\end{array}$ & $\begin{array}{l}\text { nenua } \\
\text { le-OBV } \\
\text { et Basile) }\end{array}$ \\
\hline
\end{tabular}

Dans ce cas, ce sont les enfants et ce qu'ils font qui est mis en relief dans le déroulement du récit. Par contre dans le cas suivant, c'est le lièvre qui est présenté comme plus important, même s'il est en position de complément d'objet du verbe:

$\begin{array}{llllr}\text { Minuât } & \text { kue } & \text { itukut } & \text { nenua } & \text { nâpessa: }[\ldots] \\ \text { Á nouveau } & \text { et } & \text { dire-3 } \mathrm{OBV}>3 \mathrm{~s} & \begin{array}{l}\text { les-OBV } \\ \text { garçons-OBV }\end{array} \\ \text { "Et à nouveau les garçons } & \text { lui disent: }[\ldots] "(\mathrm{McN} \text { (...ty et Basile) }\end{array}$

L'obviatif est important dans la construction textuelle en ce qu'il sert à avertir les auditeurs ou les lecteurs d'un changement de statut des personnages dans le déroulement des événements. Ainsi un nom obviatisé dans une phrase et qui devient proche dans la phrase suivante indique que cet acteur va devenir ou redevenir actif au niveau du déroulement des événements. Le changement de statut grammatical d'obviation constitue donc une invitation explicite à redoubler d'attention quant au rôle qu'un participant joue dans un événement. Il est certainement très difficile de traduire cette dimension dans le passage d'une langue algonquienne vers une langue indo-européenne.

\subsection{Les ordres $d u$ discours: dimension textuelle et rhétorique}

Les "ordres" constituent aussi une catégorie grammaticale propre aux langues algonquiennes (Cyr 1991). Cette catégorie est exprimée exclusivement dans le verbe, bien que son emploi puisse avoir une influence sur les autres parties du discours.

De la même manière que le rôle de l'obviation consiste à indiquer l'importance d'un participant, le rôle des ordres est d'indiquer l'importance accordée à une action, ou à un événement dans le déroulement global d'un récit. Ainsi, la langue innu-montagnaise met à disposition trois séries de morphèmes particuliers, appelés les ordres du discours ${ }^{3}$ : "l'indépendant", le "conjonctif simple" et le conjonctif de "forme changée", permettant de distinguer l'importance des événements dans le cadre d'un discours ou d'un récit. Voici un exemple des différentes formes que peut prendre un verbe, selon qu'il se présente à l'indépendant ou à l'une des deux subdivisions du conjonctif, c'est-à-dire le conjonctif simple ou la forme changée:

$\begin{array}{ll}\text { INDÉPENDANT } & \text { nua:pama:u } \\ & \text { tshua:pama:u } \\ & \text { ua:pameu }\end{array}$

'je le vois'

'tu le vois'

ua:pameu

'il le voit' 


$\begin{array}{lll}\text { CONJONCTIF SIMPLE } & \text { ua:pamak } & \text { 'je le vois' } \\ & \text { ua:pamat } & \text { 'tu le vois' } \\ & \text { ua:pama:t } & \text { 'il le voit' } \\ \text { FORME CHANGÉE } & \text { uia:pamak } & \text { 'je le vois' } \\ & \text { uia:pamat } & \text { 'tu le vois' } \\ & \text { uia:pama:t } & \text { 'il le voit' }\end{array}$

L'indépendant se distingue du conjonctif par la forme que prennent les marques personnelles. On voit que l'indépendant est marqué par des désinences personnelles en plus d'un préfixe personnel à la première et à la deuxième personne. Au conjonctif et à la forme changée il n'y a pas de préfixe. Par contre les désinences diffèrent d'une personne à l'autre et ce à toutes les personnes. Enfin, la forme changée se distingue du conjonctif simple par une altération de la voyelle de la première syllabe du radical. Ces exemples, isolés de tout contexte, ne disent pas grand-chose sur la différence de sens que produisent les ordres dans la totalité d'un discours. Pour mieux les comprendre, essayons d'abord de saisir leur fonction pour ensuite les regarder à l'oeuvre dans un court texte.

L'ordre indépendant indique que le verbe pourvu de ce morphème exprime une situation considérée comme faisant partie de l'arrière plan de la narration. En général on trouve à l'indépendant, dans les récits et discours montagnais, les verbes exprimant la présentation du récit, le lieu, l'époque, les verbes signalant l'existence des personnages, citant leur nom et leurs caractéristiques fondamentales, etc. Dans la suite, les verbes à l'indépendant viendront, à intervalle régulier, meubler le récit de détails explicatifs relatifs aux actions et au comportement des personnages, sur leurs réflexions et leurs états d'âme, etc.

Les morphèmes d'ordre conjonctif, quant à eux, indiquent que les verbes ainsi marqués expriment les événements réels et les événements marquants du récit, ceux qui sont considérés comme importants par le narrateur (et qui doivent l'être aussi pour les auditeurs). Les fonctions des deux formes du conjonctif se répartissent comme suit:

- la forme changée sert à mettre en relief l'entrée en scène des personnages, les événements déclencheurs des actions réelles ou les situations qui annoncent les événements fondamentaux du récit, autrement dit tout ce qui constitue un facteur de changement dans l'ordre connu des choses.

- le conjonctif simple, pour sa part, exprimera les événements fondamentaux, c'est-à-dire ceux qui constituent, dans l'ordre de leur déroulement réel, les faits et gestes des personnages.

Autrement dit, l'indépendant constitue l'arrière-plan, le décor esquissé par le narrateur, tandis que le conjonctif projette sur le devant de la scène les acteurs et leurs actions.

Voyons maintenant la traduction française d'un extrait d'un récit (McNulty et Basile) illustrant la façon dont s'articulent les ordres du montagnais. Une façon de traduire l'importance rhétorique des ordres du dis- 
cours tels qu'ils apparaissent dans le texte de départ serait d'accorder à chacun une typographie différente. L'indépendant est en police 10, le conjonctif simple en police 12 et la forme changée en police 14 et caractères gras.

1. Autrefois, jadis, il n'y avait ni feu ni filet;

2. c'est alors que le Mehapuh part pour voir comment on faisait du feu et comment on fabriquait des filets.

3. Et Mehapuh court jusqu'à la mer.

4. Arrivé là cependant...

5. Que faire? Il sait nager mais il ne peut pas traverser car la mer est trop large.

6. Ensuite il appelle des baleines: "Alignez-vous baleines, je veux traverser la mer!"

\section{Alors vraiment viennent s'aligner des baleines,}

8. et ensuite il saute de l'une à l'autre Mehapuh.

On voit que la phrase 1, dont le verbe est à l'indépendant, sert à introduire l'époque et le décor dans lequel va se dérouler le récit. La phrase 2, à la forme changée, exprime l'événement qui fait démarrer l'action réelle: Méhapuh décide de partir à la recherche du feu et des filets de pêche. Puis, avec la phrase 3, l'action réelle commence: Méhapuh part en courant vers la mer. En 4 on signale un obstacle qui va encore une fois initier (=forme changée) un changement dans le cours des choses. La phrase 5 vient nous donner des détails (= indépendant) sur la nature de cet obstacle. À la phrase 6 l'action réelle reprend mais la phrase 7 apporte un nouveau changement dans l'état du monde où se déroule ce récit. Ce nouvel état des choses initie une nouvelle séquence d'événements énoncés en 8: il se met à sauter de l'une à l'autre.

Un locuteur peut, dans une certaine mesure, choisir l'ordre selon lequel il énoncera les événements. S'il souhaite indiquer à ses allocuteurs que tel événement joue un rôle prépondérant dans la suite de son récit, il mettra le verbe relatant cet événement à la forme changée.

\footnotetext{
"Eukuan eshku uet apashtakan esht ne matutisha:n."

"C'est vraiment à cause de ça qu'on pratique encore la suerie."

(Bacon \& Vincent 1979)
}

Ici, le ça réfère aux paroles d'un certain Anglais qui aurait, dit-on, encouragé ouvertement les Montagnais à continuer de faire usage de la tente à suerie. Fort de cette caution donnée par l'Anglais, le vieux narrateur montagnais va ensuite raconter comment les Montagnais pratiquent cet usage. Ici, la mise en exergue de la caution donnée par le riche voyageur anglais sert à 
masquer la responsabilité des Montagnais eux-mêmes face à l'usage de la suerie. Ceci se comprend, lorsqu'on sait que les prêtres à l'époque interdisaient formellement un tel usage qui relevait selon eux de la sorcellerie.

Par contre, si l'on souhaite, pour des raisons personnelles ou contextuelles, diminuer l'impact de ce qu'on va dire, on met le verbe à l'indépendant, mode de l'arrière-plan ou, pour ainsi dire, de la mise en veilleuse.

$\begin{array}{llll}\text { "Ma:te iat passe } & \text { tshishenuat } & \text { eshk } & \text { mastutisheuat." } \\ \text { "Ainsi de nos jours } & \text { des vieux } & \text { encore } & \text { font suerie." } \\ \text { (Bacon \& Vincent 1979) } & & \end{array}$

Ici, le locuteur donne cette information comme une information brute et non pas comme un fait important de son récit.

Enfin, les faits réels, importants, et dont il convient absolument de se souvenir, sont présentés au conjonctif. Par exemple, lorsqu'on cite les paroles de quelqu'un et qu'on veut réellement insister sur l'importance de ce qui est dit, on mettra le verbe dire au conjonctif. Dans l'exemple suivant, le verbe au conjonctif (en police 12) met en lumière l'importance des paroles citées, tandis que le second verbe dire sous la forme itanu, à l'indépendant en police 10, indique simplement que tout ce qui précède a été rapporté au narrateur; autrement dit, il ne l'a pas entendu lui-même:

(11) "Ekue itat: "Eukuan mestamenuakue nikan matutishân." itanu.

"Et il lui dit: "Voilà que je me rends compte que c'est très bon la tente à suerie," dit-on.

Cette spécificité morphologique à portée textuelle, combinée à l'usage quasi exclusif du discours direct, donne à la structure de l'argumentation algonquienne une finesse et une subtilité qui ne peuvent être traduites intégralement qu'au moyen de stratégies explicatives et visuelles qui ne sont pas toujours disponibles ou utilisables dans le produit traduit.

\subsection{Le discours direct : langue et tradition orale}

Le montagnais est une langue qui répugne à l'usage du discours indirect. En français, par exemple, si quelqu'un vous dit qu'un de ses amis lui a dit: “Je vais venir demain avec mon chien", vous pouvez rapporter cette information en y ajoutant du vôtre et dire à votre tour à quelqu'un d'autre: "Il a dit qu'il viendrait demain avec son fichu chien!'”. En montagnais par contre l'usage et le style exigent que l'on ne change pas ainsi l'information. C'est pourquoi il faut toujours rapporter directement les paroles de quelqu'un, sans rien y changer et indiquer aussi fidèlement que possible la provenance de cette information. Dans un article traitant de cette notion du respect des sources de l'information, Drapeau (1985: 27) rapporte l'exemple suivant: 


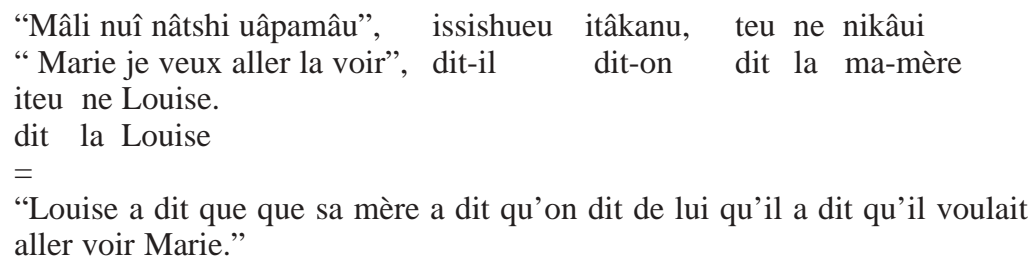

Voyons maintenant à partir d'un texte montagnais, La suerie, idéologiquement marqué, dans quelle mesure la combinaison des ordres du discours et l'usage du discours direct rapporté marquent les fondements de la rhétorique algonquienne, souvent imperceptible pour les Occidentaux ou, si elle est perçue, reste loin d'être systématiquement traduisible.

Ce récit s'étale sur cinq paragraphes et contient 132 verbes dont les formes se répartissent comme suit: 41 conjonctifs simples, dont 14 sont des verbes qui introduisent les paroles d'autrui, 41 formes changées et 50 indépendants, dont 24 verbes introducteurs. Les référents en position de sujets sont variés: un certain Anglais, une vieille Montagnaise, le narrateur qui s'inscrit comme un je, certains vieux Montagnais qui sont morts à l'époque de la narration, la femme du narrateur et, enfin, des référents anonymes exprimés par on; il est difficile de décider si le narrateur se fond dans ce on ou s'il s'en dissocie. Nous croyons qu'il s'en dissocie, bien que d'autres puissent ressentir le contraire. Cela dépend de la façon dont ce discours particulier résonne dans l'inconscient de chaque lecteur.

Voyons d'abord la version française du texte innu. Nous avons ajusté la typographie des propositions en fonction de l'importance discursive et rhétorique qui leur était accordée dans la grammaire du texte original. On trouvera donc des fragments de texte en police 14 en caractères gras, police 12 et police 10. Le contexte socioculturel dans lequel le texte original a été produit est le suivant: enregistré au début des années soixante-dix, à l'époque où les Autochtones étaient sous la coupe de missionnaires qui condamnaient toute forme de pratique rituelle ancestrale, l'auteur est interviewé par un anthropologue qui lui demande de parler des us et coutumes. On sent que l'auteur veut livrer son savoir sur la pratique de la suerie, mais en même temps, il ne veut pas encourir le blâme du prêtre. Il va donc enrober son discours de précautions oratoires emphatiques à propos d'un Anglais qui aurait fortement incité les Innus à continuer cette pratique. Et lorsque l'auteur-narrateur se présente lui-même comme un acteur du rituel de la suerie, il se présente littéralement caché stylistiquement derrière sa femme et à l'article de la mort.

\section{LA SUERIE}

C'est ce qu'il-lui-a-dit l'Anglais après qu'il-lui-ait-parlé. Ensuite l'Anglais fit-faire une tente à suerie, ensuite il-y-fit-entrer sa femme, ensuite il-l'appela la... une... la vieille-femme celle-là, dit-on, ensuite elle-s'assit-àcôté de sa femme. Alors donc elle-lui-souffla-sur dit-on toute la peau, elle-souffla-à-répétition et elle-lui-sécha-la-peau entièrement avec un 
linge, elle-changea le linge, ensuite à-nouveau elle-lui-sécha-la-peau, dit-on, la femme, jusqu'à ce qu'elle sorte. Ensuite elle-sortit, dit-on. Ensuite onn'eut-pas-à-recommencer. C'est vraiment cela. Après il-fut-trèscontent, dit-on, il-fut-très-content- de cela, dit-on. Ensuite il lui dit à l'homme, lui-parlant-ainsi: "Je me rends compte que c'était très bon, ce que tu-m'as-dit," lui-dit-il ensuite, dit-on, "c'est très bon ce que tu m'as dit" lui-dit-il ensuite, dit-on; 'c'cest vraiment quelque chose que vous ne devrez jamais abandonner" lui-dit-il ensuite l'Anglais dit-on, "cela lui a fait du bien de me l'avoir fait suer ainsi"' lui-dit-il ensuite, dit-on 'c'est que je me rends compte que c'est très bon la suerie"' lui-dit-il ensuite, diton "pour moi elle était pratiquement morte ma femme" lui-dit-il ensuite, dit-on. Ensuite il-raconta-cela à sa femme [et] elle-s'adressa-en-anglais à la montagnaise: "je-te-remercie-beaucoup" medit-elle; ensuite elle-lui-dit la femme (montagnaise): "Tellement il était content qu'ensuite il-me-donna cinquante dollars" dit-elle ensuite, dit-on. "Je ne voulais presque pas le prendre." Ensuite il-lui-dit, dit-on: "Mais moi aussi j'ai-pitié-de-toi." me-dit-il. Ensuite il-lui-dit, dit-on "Vraiment je-te-remercie", me-dit-il, lui dit-il ensuite, dit-on.

Comme on le voit, la seule façon de rendre en français l'importance discursive des événements et des citations tels qu'exprimés par la langue innu est d'utiliser la stratégie des diverses polices de caractères ainsi que le gras. Le seul usage des temps et des modes verbaux français ne saurait ni restaurer la scénographie du texte original, ni restituer le jeu des acteurs.

\subsection{Familles de mots et familles de sens: langue et vision du monde}

Lorsqu'on traduit d'une langue à l'autre, on part d'un mot dans une langue et on arrive à un mot ou à une périphrase dans une autre. C'est un peu comme si on prenait un concept dans une langue et qu'on l'amenait à migrer vers une autre langue en abandonnant la famille conceptuelle à laquelle le mot d'origine appartenait. Par exemple, étant donné le mot mìgmaq très concret baqtesm 'loup', en traduisant on détache la notion de toutes les notions apparentées par la forme baqt-, entre autres baqtaqawàq 'l'écho d'un son qui résonne et se répète'.

On dit parfois que l'on ne peut vraiment bien connaître quelqu'un que si on le rencontre parmi les siens. Nous croyons qu'il en va de même des mots. C'est ce qui fait dire au philosophe J. Sakej Henderson (1993: p.c.):

La langue mìgmaq a toujours été composée de forces (mntu'k) qui sous-tendent la réalité perçue - qui lui donnent naissance, la transcendent, l'actualisent et la transforment. Toutes ces forces peuvent changer de forme, de contenu, mais collectivement elles sont créatrices de dignité. [...] Le processus est 
plus qu'une définition des phonèmes ou des sons, des mots et des phrases. [...] Tel que représenté par la structure de la langue, dans un monde interconnecté, le tout cesse d'être le tout lorsqu'il devient part d'une explication. Un processus holistique ne peut pas être expliqué si on le fracasse d'abord en ses multiples parties constituantes, pour assigner ensuite des explications locales à ces divers constituants. (Trad. par les auteurs)

\section{Tendances traductologiques}

Dans cette section, nous discutons de la nature des textes qui ont été traduits de l'anglais au mìgmaq, une autre langue algonquienne, mais du groupe oriental cette fois. Nous tenterons aussi de faire un tour d'horizon des tendances traductologiques prévalentes dans le monde des gens lettrés mìgmaqs.

\subsection{Difficultés logistiques: la question des nombreux systèmes d'écriture}

La nation mìgmaq est aujourd'hui dispersée sur dix-neuf réserves chevauchant le Québec, le Nouveau Brunswick, la Nouvelle Écosse, Terre-Neuve et le Maine, aux Etats-Unis. Au niveau de l'écriture, les représentants des diverses communautés ne disposent pas d'une orthographe commune ce qui nuit à la production littéraire et à la publication de textes. Par exemple, une publication réalisée dans une communauté donnée peut être difficilement diffusée dans une autre. La rareté des lecteurs à l'intérieur d'un sous-système orthographique n'incite guère à engager de lourdes dépenses pour une publication. Et la rareté des productions écrites décourage à son tour les efforts d'alphabétisation.

De plus, les discussions autour de la manière d'écrire un mot sont parfois si longues et si pénibles qu'on s'épuise totalement à la préparation de manuscrits et souvent on finit par ne pas les publier. Même si on publie un manuscrit, son orthographe devient périmée en moins de temps qu'il ne faut pour épuiser la vente ou l'exploitation de l'ouvrage. Enfin, à cause de la vie souvent courte des Conseils de bandes dont les mandats sont renouvelables tous les deux ans, il arrive trop souvent qu'un ouvrage, préparé sous un gouvernement, ne soit plus prévu à la publication sous un autre.

\subsection{Le Nouveau Testament en langue mìgmaq: paradoxe de la traduction}

Parmi les oeuvres traduites récemment, une des plus imposantes est certainement le Gelulg Glusuaqan ou Nouveau Testament en langue mìgmaq du pasteur Baptiste Watson Williams (1999). Cet ouvrage comprend les Évangiles, les Actes des Apôtres, les Épîtres et le texte de la Révélation. En tout, un document de huit cents pages incluant les textes en traduction mìgmaq et 
la version de départ anglaise. La tâche a requis du traducteur une somme de 712 semaines, c'est-à-dire presque quatorze ans de travail à temps complet. Outre les trois traducteurs mìgmaq Emmanuel Metallic, Nellie Wysote et Marion Wilmot, toute une myriade de consultants Mìgmaq ont été invités à apporter leur contribution sous forme de critiques, opinions, conseils et commentaires. En fin de compte, il s'agit d'une oeuvre magistrale et d'une banque de mots formidable. Mais le fait est qu'aujourd'hui, parmi les ressortissants mìgmaq, à peine trente pour cent ont encore la langue orale comme langue d'usage, et parmi ceux-ci à peine une poignée des locuteurs sont suffisamment alphabétisés pour pouvoir lire la langue avec un degré d'aisance raisonnable. On peut donc conclure que le Gelulg Glusuaqan ne sera pratiquement pas lu, sauf occasionnellement et par petits extraits lors de cérémonies religieuses. Il faut aussi conclure que de telles oeuvres servirontelles beaucoup plus à produire du corpus, qu'à actualiser la langue. Mais là encore, il y a problème. En effet, les commentaires de certains Mìgmaq lettrés semblent indiquer que la langue du Gelulg Glusuaqan, bien que parfaitement grammaticale, utiliserait un style "évangélique" inexistant dans leur langue. Somme toute, bien que les lettrés Mìgmaq reconnaissent la valeur de l'oeuvre, ils hésiteraient à l'utiliser comme corpus représentatif et garant de leur langue ancestrale. Il reste donc difficile de juger de la valeur traductologique de l'oeuvre puisque très peu de lecteurs potentiels sont assez qualifiés pour en faire un compte-rendu détaillé.

\subsection{Nouvelles tendances}

Ainsi, on se retouve avec un corpus assez volumineux et dont la partie en langue originale amérindienne est encore partiellement manuscrite; il s'agit majoritairement de matériau pédagogique de fabrication artisanale ou de transcription de corpus oraux, et de traductions à partir de textes anglais ou français. Le monde mìgmaq est à cet égard assez représentatif de ce qui se passe dans le monde amérindien en général. La situation, en constante évolution, s'ouvre cependant à de nouvelles solutions.

\subsubsection{Electronic Encyclopedic Dictionary of Contemporary Migmaq: une tentative de transmission par la traduction}

Un exemple de cette évolution concerne le Electronic Encyclopedic Dictionary of Contemporary Mìmaq (Cyr, Metallic, Sévigny). Nous avons décidé d'adopter une orthographe phonétique - le système Metallic - qui n'a pas de parti-pris géo-politique, n'étant utilisé comme orthographe officielle dans aucune communauté. Son orthographe est la première à avoir été créée par un linguiste mìgmaq, Emmanuel Metallic. Nous espérons que cet orthographe pourra devenir une sorte de scripta franca, une idée dont nous discutons ailleurs (Cyr \& Sévigny 2003). Le dictionnaire présente aussi, pour la première fois, la rédaction d'entrées faite entièrement du point de vue cultu- 
rel et pragmatique de la culture mìgmaq. Le fait que les entrées devaient figurer en anglais nous a posé un problème: comment créer un document qui permettrait de transmettre la culture et la langue mìgmaq à une nouvelle génération de locuteurs sans changer de façon intégrale la nature de cette culture? Comment aider des adultes et des jeunes qui ont perdu leur langue à la redécouvrir, sans la réinventer à travers la lentille de l'anglais? La solution que nous avons fini par adopter a privilégié les structures et la cadence du mìgmaq dans l'anglais des entrées, même si cette option entrânait des difficultés éditoriales du point de vue de l'anglais. Nous avons choisi, par exemple, de situer les entrées dans l'espace discursif centré sur le "tu", et nous avons ainsi présenté tous les verbes du dictionnaire à la deuxième personne comme personne matrice. Ainsi, nous proposons de faire prévaloir la spécificité de l'organisation conceptuelle du mìgmaq dans notre dictionnaire. Nous espérons que les locuteurs du mìgmaq pourront arriver à reconstituer un espace culturel et linguistique qui leur sera familier, bien qu'il soit presque entièrement réalisé par le biais de la langue coloniale.

\subsubsection{Intellectualité et traduction: les intellectuels réincarnateurs}

Depuis les années 1990, on assiste à une augmentation des publications mìgmaq en anglais surtout, mais en mìgmaq aussi, et dont l'essentiel prend naissance dans la réserve d'Eskasoni en Nouvelle-Écosse avec quelques ramifications à Listuguj au Québec, Big Cove au Nouveau Brunswick et Chapel Island en Nouvelle Écosse. On peut dire que ces quatre communautés constituent les foyers de l'intellectualité mìgmaq, dont les principaux artisans sont, entre autres, Emmanuel Metallic, Marie Battiste, Rita Joe, Murdena Marshall, Katherine Sorbey, Helen Sylliboy, Stephen Augustine, Noel Knockwood, Lindsay Marshall, Daniel Paul et par adoption, J. S. Youngblood Henderson.

Les publications, dans le domaine de la poésie, de l'histoire et de la spiritualité/philosophie sont de plus en plus importantes et régulières. De tous ces auteurs, seules Katherine Sorbey (Usmiani et al. 1994: 88-89), Rita Joe (1996: 10) et Lindsay Marshall (1997: 52-53) ont produit des textes originaux en mìgmaq avec une traduction en anglais. Mais d'autres se disent déterminés à poursuivre la tendance. En particulier Helen Sylliboy et Lindsay Marshall qui nous annoncent que leurs prochaines oeuvres seront entièrement en mìgmaq avec traduction anglaise.

\subsubsection{L'intraduisible dans le traduit: les mots mìgmaq dans l'anglais amérindien}

De plus en plus, les auteurs amérindiens qui écrivent en anglais utilisent cependant très librement les mots mìgmaq pour compenser l'intraduisible. Les poèmes de Rita Joe ou de Lindsay Marshall, entre autres, nous fournissent de nombreux exemples du procédé. 


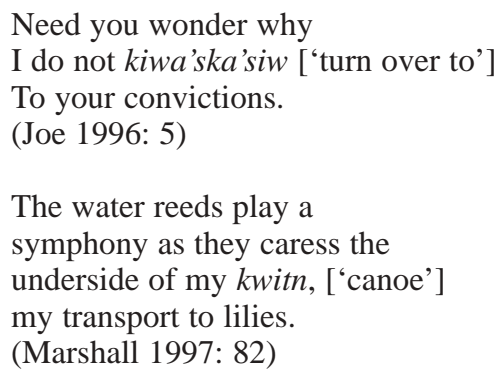

Cette tendance se retrouve dans tous les types de textes, y compris les articles scientifiques ou les écrits philosophiques. À cet égard, la démarche la plus remarquable est celle de James Sakej Youngblood Henderson dans le récent ouvrage The Mikmaw Concordat (1997). Les numéros de chapitres et la majeure partie de la table des matières sont en mìgmaq. De même, à l'intérieur du texte un nombre important de termes philosophiques sont en mìgmaq. Un glossaire bilingue est fourni en annexe précédé d'un avertissement aux lecteurs (p.116):

In Mìkmaw speech, a person's speech pattern reflects his or her mood and determines the meaning of the sounds. The printed word cannot capture the diversity of patterns or meaning. Thus in many situations, one cannot determine the actual translations.

\section{Conclusion}

Ce mouvement d'appropriation de la langue dominante teintée de couleurs lexicales amérindiennes constitue en quelque sorte une actualisation de la notion de "middle ground", un terrain de rencontre qui permet aux usagers à la fois d'éviter les écueils les plus onéreux de la traduction classique et de se retrouver comme société dans les textes, qu'ils soient destinés aux co-sociétaires ou aux étrangers.

\section{Bibliographie}

\section{Sources primaires}

Joe, Rita (1978). Poems of Rita Joe. Halifax, N.S.: Abanaki Press.

Joe, Rita (1996). Song of Rita Joe: autobiography of a Mi'kmaq poet; with the assistance of Lynn Henry. Charlottetown, PEI: Ragweed Press.

Joe, Rita et Lesley Choyce (1997). The Mi'kmaq Anthology. Lawrencetown Beach: Pottersfield Press.

Marshall, Chief Lindsay (1997). Clay Pots and Bones Pka'wo'qq aq Waqntal. Halifax: Solus Publishing. 


\section{Sources secondaires}

Bacon, Pipin \& Sylvie Vincent (éds) (1979). Atanutshe Nimushum, récits racontés et recueillis par les montagnais de Natashquan. Édition française et montagnaise. Québec: Bacon et Vincent.

Clarke, Sandra (éd.) (2003), PAMAPLA 26. St. John's, NL: Memorial University Press.

Cowan, William (éd.) (1991). Papers of the Twenty-second Algonquian Annual Conference. Ottawa: Carleton University Press.

Cyr, Danielle (1991). "Orders as Aspectual Markers in Montagnais: Typological Evidence and Pragmatic Considerations." W. Cowan (éd.) (1991). Papers of the Twenty-second Algonquian Annual Conference. Ottawa: Carleton University Press, 20-49.

Cyr, Danielle (1996). "La lune est notre grandmère: la notion de vérité comme principe assimilateur." D. Daviault \& J.Mailhot (éds) (1996). Recherches amérindiennes au Québec, 26, 43-51.

Cyr, Danielle, Metallic, Emmanuel \& Alexandre Sévigny (à paraître). Electronic Encyclopedic Dictionary of Contemporary Migmaq. Québec: Presses de l'Université Laval.

Cyr, Danielle \& Alexandre Sévigny (2003). "Critically examining the construction of a endangered language dictionary." S. Clarke (éd.) (2003). PAMAPLA 26. St. John's, NL: Memorial University Press, 91-104.

Daviault, Diane \& José Mailhot (éds) (1996). Recherches amérindiennes au Québec, $26,4$.

Drapeau, Lynn (1985). "Le traitement de l'information en montagnais." Recherches amérindiennes au Québec XIV (4), 24-33.

Henderson, Sakej (1993). "Governing the Implicate Order: Self-Government and the Linguistic Development of Aboriginal Communities." Communication présentée au congrès Linguistic Justice for Aboriginal People in Canada, 5 novembre 1993. Ottawa: Université d'Ottawa, Faculté de Droit.

Henderson, Sakej (1997). The Mikmaw Concordat. Halifax: Fernwood.

McNulty, Gerry et Basile, Marie-Josée. (manuscrit n.d.). Mehapuh natshiskuteu. U. Laval: Département d'anthropologie.

Usmiani, Renate et al. (1994). Kelusultiek: original women's voices of Atlantic Canada. Halifax: Institute for the Study of Women, Mount Saint Vincent University.

Williams, Watson (1999). Gelulg Glusuaqan Gisiteget Agnutmugsi'gw. Toronto: Canadian Bible Society.

Wolfart, Christopher. (1973). "Plains Cre: A Grammatical Study." Transactions of the American Philosophical Society 63 (5), 5-90.

1 Le terme algonquin réfère à l'ensemble de toutes les nations de la famille algonquienne, incluant entre autres les Cris, les Innus, les Attikamek, les Algonquiens, les Mìgmaq, etc. Par opposition, le terme algonquien réfère plus spécifiquement à la nation algonquienne.

2 Dans cette discussion il est de première importance de distinguer entre pronoms personnels et personnes verbales. Les pronoms personnels sont des morphèmes grammaticaux qui remplacent les noms. Les personnes verbales sont des notions sémantiques et cognitives qui réfèrent aux acteurs des situations exprimées par les verbes.

${ }^{3}$ Cette terminologie a été utilisée par Bloomfield d'abord et par tous les algonquianistes à sa suite. Pour une explication plus élaborée, voir, entre autres: Wolfart (1973). 